\title{
STABILITY OF STEADY STATES IN KINETIC FOKKER-PLANCK EQUATIONS FOR BOSONS AND FERMIONS*
}

\author{
LUKAS NEUMANN $^{\dagger}$ AND CHRISTOF SPARBER ${ }^{\ddagger}$
}

\begin{abstract}
We study a class of nonlinear kinetic Fokker-Planck type equations modeling quantum particles which obey the Bose-Einstein and Fermi-Dirac statistics, respectively. We establish the existence of classical solutions in the perturbative regime and prove exponential convergence towards the equilibrium.
\end{abstract}

Key words. Fokker-Planck operator, Bose-Einstein statistics, Fermi-Dirac statistics, long time behavior

AMS subject classifications. 82C40, 82C10, 76R50, 82D 37

\section{Introduction and main results}

In recent years the rigorous mathematical study of kinetic equations has been enlarged to a class of models which take into account also quantum effects, cf. [25] for a general overview. This so-called quantum kinetic theory can be seen as an attempt to incorporate certain properties of an underlying quantum system into the framework of classical statistical mechanics. One might hope that these "hybrid models" on the one hand allow for a somewhat simpler description of the particle dynamics, while maintaining, on the other hand, some purely quantum mechanical features such as generalized statistics for Bosons and Fermions. Clearly such models can only be justified in a semi-classical regime, respectively, in situations where the transport properties of the particles are mainly governed by Newtonian mechanics. Indeed, this point of view has already been adopted in the classical paper by Uehling and Uhlenbeck [24], in which they derived their celebrated nonlinear Boltzmann type equation for quantum particles.

Following their spirit most of the quantum kinetic models studied so far invoke nonlinear collision operators of Boltzmann type, see, e.g., [5, 6, 16, 17]. For these kind of models, a focus of interest is on the long time behavior of their solutions, in particular the convergence towards steady states, which generalize the classical Maxwellian distribution, $c f$. $[8,16,18,20]$. Very often though, the simplified case of a spatially homogeneous gas is considered.

In the present work, we shall also be interested in such relaxation-to-equilibrium phenomena, in the spatially inhomogeneous case. We shall not deal with a Boltzmann type equation, but rather study a nonlinear Fokker-Planck type model (FP). More precisely, we consider

$$
\partial_{t} f+p \cdot \nabla_{x}\left(f+\kappa f^{2}\right)=\operatorname{div}_{p}\left(\nabla_{p} f+p f(1+\kappa f)\right),
$$

where, for any $t \geq 0, f=f(t, x, p) \geq 0$ denotes the particle distribution on phase space $\Omega_{x} \times \mathbb{R}_{p}^{d}$. In what follows, the spatial domain is chosen to be $\Omega_{x}=\mathbb{T}^{d}$, the $d$ dimensional torus. This setting can be seen as a convenient and mathematically

\footnotetext{
${ }^{*}$ Received: January 16, 2007; accepted (in revised version): July 27, 2007. Communicated by Lorenzo Pareschi.

${ }^{\dagger}$ Institute for Analysis and Scientific Computing, Technical University Vienna, Wiedner Hauptstr. 8, A-1040 Vienna, Austria (lukas.neumann@tuwien.ac.at).

${ }^{\ddagger}$ Wolfgang Pauli Institute Vienna \& Faculty of Mathematics, Vienna University, Nordbergstraße 15, A-1090 Vienna, Austria (christof.sparber@univie.ac.at). C. Sparber has been supported by the APART research grant funded by the Austrian Academy of Sciences.
} 
simpler replacement for the incorporation of confining potentials $V(x)$ needed to guarantee the existence of nontrivial steady states in the whole space. In (1.1) we set $\kappa=-1$ for Fermions and $\kappa=1$ for Bosons. For $\kappa=0$ equation (1.1) simplifies to the classical linear Fokker-Planck equation (or Kramer's equation) on phase space. For this linear model, the convergence to equilibrium has recently been studied in $[4,11,19]$, using several different approaches.

The FP type model (1.1) has been introduced in [14], for classical particles obeying an exclusion principle. A formal derivation from a generalized Boltzmann equation for fermions and Bosons is given in [12], and from the Uehling-Uhlenbeck equation in [21]. Different physical applications can be found in $[9,13,15]$ dealing with, both the spatially homogeneous as well as the inhomogeneous case (see also [10] and the references therein). More recently a similar but somewhat simpler FP type model has been proposed in $[22,23]$ to describe self-gravitating particles and the formation of Bose-Einstein condensates in a kinetic framework. The authors consider

$$
\partial_{t} f+p \cdot \nabla_{x} f=\operatorname{div}_{p}\left(\nabla_{p} f+p f(1+\kappa f)\right),
$$

where, in contrast to (1.1), only the diffusive part of the equation includes a nonlinearity. As we will see, however, both equations share the same steady states. In order to deal with both models at the same time we study from now on the following initial value problem:

$$
\left\{\begin{array}{l}
\partial_{t} f+p \cdot \nabla_{x}\left(f+\sigma \kappa f^{2}\right)=\operatorname{div}_{p}\left(\nabla_{p} f+p f(1+\kappa f)\right), \\
\left.f\right|_{t=0}=f_{0}(x, p)
\end{array}\right.
$$

with $\sigma=1$, or $\sigma=0$, corresponding to the case (1.1) and (1.2), respectively. We note that the long time behavior of these models in the spatially homogeneous case (and for $d=1$ ) has been rigorously investigated quite recently in [3] via an entropy-dissipation approach.

In what follows, the initial phase space distribution $f_{0} \in L^{1}\left(\mathbb{T}_{x}^{d} \times \mathbb{R}_{p}^{d}\right)$ is assumed to be normalized according to

$$
\iint_{\mathbb{T}^{d} \times \mathbb{R}^{d}} f_{0}(x, p) \mathrm{d} x \mathrm{~d} p=M
$$

for some given mass $M>0$. This normalization is conserved by the evolution. Moreover in the fermionic case, i.e. $\kappa=-1$, we require $f_{0}(x, p)<1, \forall(x, p) \in \mathbb{T}^{d} \times \mathbb{R}^{d}$, as usual in the physics literature [9]. In particular the latter is needed to define the associated quantum mechanical entropy functional, i.e.

$$
H[f]:=\iint_{\mathbb{T}^{d} \times \mathbb{R}^{d}}\left(\frac{|p|^{2}}{2} f+f \ln f-\kappa(1+\kappa f) \ln (1+\kappa f)\right) \mathrm{d} x \mathrm{~d} p,
$$

which obviously requires $f(t, x, p)<1$, if $\kappa=-1$. It is now straightforward to verify that (independent of the particular choice of $\sigma$ ) the unique steady state of (1.3) is given by

$$
f_{\infty}=\frac{1}{\exp \left(\frac{|p|^{2}}{2}+\theta\right)-\kappa}
$$

where the constant $\theta$ is used to ensure that $f_{\infty}$ satisfies the mass constraint (1.4). In the Bosonic case we require $\theta \in \mathbb{R}_{+}$, whereas in the fermionic case we can allow 
for $\theta \in \mathbb{R}, c f$. $[3,7]$ for more details. In the latter situation the distribution (1.5) is the well known Fermi-Dirac equilibrium distribution. On the other hand, for $\kappa=1$, $f_{\infty}$ is the so-called regular Bose-Einstein distribution. Finally, if $\kappa=0$, formula (1.5) simplifies to the classical Maxwellian, i.e.

$$
f_{\infty}^{\operatorname{lin}}=\frac{M}{(2 \pi)^{d / 2}} e^{-|p|^{2} / 2},
$$

where $\log \left(M(2 \pi)^{-d / 2}\right)=-\theta$. Note that in any case the equilibrium state is independent of $x$ since we have chosen our spatial domain to be $\mathbb{T}^{d}$.

In the Bosonic case there is an additional difficulty, at least for $d \geq 3$, since one can not associate an arbitrary large $M>0$ to the steady state. More precisely, the maximum amount of mass comprised by $f_{\infty}$ is determined via

$$
\iint_{\mathbb{T}^{d} \times \mathbb{R}^{d}} \frac{1}{e^{|p|^{2} / 2}-1} \mathrm{~d} x \mathrm{~d} p=: M_{\text {crit }}<\infty,
$$

i.e. for $\theta=0$. Due to mass conservation this induces a threshold on $M$. This problem, which does not appear in dimensions $d=1$ or 2 , has led to the introduction of more general Bosonic steady states, where an additional $\delta$-distribution (appropriately normalized) is added to $f_{\infty}, c f$. [6, 8]. This singular measure can then be interpreted as a so-called Bose-Einstein condensate (BEC). The formation of a $\delta$-measure in finite or infinite time is a task of extensive research in quantum kinetic theory, see, e.g., [2, 8]. For our nonlinear model though, including such generalized solutions on a rigorous mathematical level seems to be out of reach so far and we thus have to impose $\theta>0$ in the Bosonic case. (Indeed, as we shall see below, we also require $\theta>0$ in the fermionic case, although for different and rather technical reasons.)

Our main task here is the description of the convergence for solutions of (1.3) towards the steady state (1.5). To this end we shall conceptually follow the approach given in [19] where the trend to equilibrium is studied for a wide class of kinetic models close to equilibrium. The difference in our case is mainly that we are dealing with local nonlinearities which moreover are also allowed to enter in the transport part of the considered equation. Mathematically speaking, the approach is based on the so-called hypo-coercivity property of the linearized equation [26, 27].

We consequently linearize the solution $f$ of $(1.3)$ around the steady state $f_{\infty}$ in the form

$$
f=f_{\infty}+g \sqrt{\mu_{\infty}},
$$

where the new unknown $g(t, x, p) \in \mathbb{R}$ can be interpreted as a perturbation of the equilibrium state such that

$$
\iint_{\mathbb{T}^{d} \times \mathbb{R}^{d}} g \sqrt{\mu_{\infty}} \mathrm{d} x \mathrm{~d} p=0 .
$$

In (1.6) we use the additional (time-independent) scaling factor

$$
\mu_{\infty}:=f_{\infty}+\kappa f_{\infty}^{2},
$$

which allows for an easier description in the functional framework given below. Plugging (1.6) into (1.3), straightforward calculations formally yield the following equation for $g$ :

$$
\partial_{t} g+\left(1+2 \sigma \kappa f_{\infty}\right) p \cdot \nabla_{x} g=L(g)+Q(g) .
$$


Above, the linearized collision operator $L$ is given by

$$
\begin{aligned}
L(g) & =\frac{1}{\sqrt{\mu_{\infty}}} \operatorname{div}_{p}\left(\nabla_{p}\left(g \sqrt{\mu_{\infty}}\right)+p \eta_{\infty} g \sqrt{\mu_{\infty}}\right) \\
& =\Delta_{p} g+g\left(\frac{d}{2} \eta_{\infty}-|p|^{2}\left(\frac{1}{4}+2 \kappa \mu_{\infty}\right)\right),
\end{aligned}
$$

where we use the short hand notation $\eta_{\infty}:=1+2 \kappa f_{\infty}$. The quadratic remainder $Q$ is

$$
Q(g)=\frac{\kappa}{\sqrt{\mu_{\infty}}}\left(\operatorname{div}_{p}\left(p \mu_{\infty} g^{2}\right)-\sigma \mu_{\infty} p \cdot \nabla_{x}\left(g^{2}\right)\right) .
$$

The main result of our work is as follows.

THEOREM 1.1. Let $f_{0}$ be of the form

$$
0 \leq f_{0}=f_{\infty}+g_{0} \sqrt{\mu_{\infty}}
$$

with $\theta>0$. Moreover if $\kappa=\sigma=1$, i.e. the Bosonic case with nonlinear transport, assume that in addition $\theta>\theta^{*}$, for a certain $\theta^{*}>0$.

For $k \in \mathbb{N}$ with $k>1+d / 2$ there exists an $\epsilon_{0}>0$, such that for all $f_{0}$ with $\left\|g_{0}\right\|_{H^{k}} \leq$ $\epsilon_{0}$, the equation (1.3) admits a unique solution $0 \leq f \in C\left([0, \infty) ; H^{k}\left(\mathbb{T}_{x}^{d} \times \mathbb{R}_{p}^{d}\right)\right)$. Moreover,

$$
\left\|\mu_{\infty}^{-1 / 2}\left(f(t)-f_{\infty}\right)\right\|_{H^{k}} \leq C\left(\epsilon_{0}\right) e^{-\tau t}
$$

where $C\left(\epsilon_{0}\right)$ and $\tau$ are positive constants.

First we collect several preliminary results in Section 2. The proof of Theorem 1.1 is then given in Section 3 .

REMARK 1.1.

- For Bosons it is crucial to avoid the possible formation of a BEC by imposing $\theta>0$, since formal calculations given in [23] indicate that an analogous theorem cannot hold if $M>M_{\text {crit }}$, see also [8]. In the fermionic case the reason to impose $\theta>0$ is to guarantee $\eta_{\infty}>0$, which we will make use of several times. It might be possible to overcome this restriction for fermions by using a different approach, see [3].

- The additional requirement $\theta>\theta^{*}$ is not needed for the model (1.2), where only a nonlinear diffusion operator is present. The reason for this constraint when dealing with (1.1) is that we have to maintain a fundamental regularizing property in $x$ of the transport part, cf. Section 3 for more details. Since $\theta^{*}$ is then determined by a transcendental equation, we do not give an exact value for $\theta^{*}$ but only perform numerical experiments which indicate that $\theta^{*} \approx 0.451$.

- As already discussed in [19] one could also take into account self-consistent potentials, which stem from a coupling to Poisson's equation. The latter case might be particularly interesting in semiconductor modeling, where the fermionic FP-type equation could be used to describe the dynamical behavior of charge carriers obeying the "physically correct" equilibrium statistics.

COROLlary 1.2. Under the same assumptions as above,

$$
H[f(t)]-H\left[f_{\infty}\right] \leq C e^{-\tau t},
$$


i.e. we have exponential decay in relative entropy.

Proof. Inserting $f=f_{\infty}+\sqrt{\mu_{\infty}} g$ into $H[f]$, having in mind $\iint \sqrt{\mu_{\infty}} g \mathrm{~d} x \mathrm{~d} p=0$, we perform a Taylor expansion around the steady state $f_{\infty}$ and finally use Theorem 1.1. This yields the assertion of the corollary.

\section{Study of the linearized collision operator}

We shall now derive several properties of the linearized collision operator $L$ to be used in the proof of the main result. First note that $L$ is self adjoint on $L^{2}\left(\mathbb{R}_{p}^{d}\right)$, and, by partial integration, one obtains

$$
\langle L(g), g\rangle_{L^{2}\left(\mathbb{R}_{p}^{d}\right)}=-\int_{\mathbb{R}^{d}}\left|\nabla_{p} g+\frac{p}{2} \eta_{\infty} g\right|^{2} \mathrm{~d} p=-\int_{\mathbb{R}^{d}}\left|\nabla_{p}\left(\frac{g}{\sqrt{\mu_{\infty}}}\right)\right|^{2} \mu_{\infty} \mathrm{d} p .
$$

Thus the kernel of the non-positive operator $L$ is given by

$$
\operatorname{Ker}(L)=\operatorname{span}\left\{\sqrt{\mu_{\infty}}\right\} .
$$

Let us define the orthogonal projection in $L^{2}\left(\mathbb{R}_{p}^{d}\right)$ onto this kernel via

$$
\Pi(f):=\left(\frac{1}{\rho_{\infty}} \int_{\mathbb{R}^{d}} f \sqrt{\mu_{\infty}} \mathrm{d} p\right) \sqrt{\mu_{\infty}},
$$

where we set

$$
\rho_{\infty}=\left(\int_{\mathbb{R}^{d}} \mu_{\infty} \mathrm{d} p\right)>0
$$

for reasons of normalization. Note that this is only a projection in the momentum variable $p \in \mathbb{R}^{d}$. Motivated by (2.1) we introduce the following weighted space

$$
\Lambda_{p}:=\left\{f \in L^{2}\left(\mathbb{R}_{p}^{d}\right):\|f\|_{\Lambda_{p}}<\infty\right\},
$$

where

$$
\|f\|_{\Lambda_{p}}^{2}:=\left\|\nabla_{p} f\right\|_{L_{p}^{2}}^{2}+\left\|p \eta_{\infty} f\right\|_{L_{p}^{2}}^{2} .
$$

Here, and in what follows, we write $L_{p}^{2} \equiv L^{2}\left(\mathbb{R}_{p}^{d}\right)$ for simplicity. Moreover, we denote by

$$
\|f\|_{\Lambda}:=\|\| f\left\|_{\Lambda_{p}}\right\|_{L^{2}\left(\mathbb{T}_{x}^{d}\right)},
$$

the induced norm on phase space. Obviously the $\Lambda_{p}$-norm controls the $L_{p}^{2}$-norm for $\kappa$ nonnegative. In the fermionic case $(\kappa=-1)$, however, this is not true in general since $\eta_{\infty}$ may change sign. For our functional approach the control of $L_{p}^{2}$ via $\Lambda_{p}$ is crucial and thus we have to guarantee that $\eta_{\infty}>0$ by assumption. This implies that for $\kappa=-1$ we need to impose $f_{\infty}<1 / 2, \forall p \in \mathbb{R}^{d}$, or equivalently $\theta>0$. This certainly is more restrictive than the usual bound, i.e. $f_{\infty}<1$, used in the physics literature. In summary we require $\theta>0$ in the Bosonic case to prevent BEC and in the fermionic case to ensure that $\eta_{\infty}$ is globally bounded away from zero.

First, we obtain a Poincaré inequality for the steady state of the linearized model. 
Lemma 2.1. If $\theta>0$ then the (strictly positive and normalized) measure $\mu_{\infty} / \rho_{\infty}$ satisfies a Poincaré inequality on $\mathbb{R}_{p}^{d}$, i.e.

$$
\int_{\mathbb{R}^{d}} g^{2} \mu_{\infty} \mathrm{d} p-\frac{1}{\rho_{\infty}}\left(\int_{\mathbb{R}^{d}} g \mu_{\infty} \mathrm{d} p\right)^{2} \leq C_{\mathrm{p}} \int_{\mathbb{R}^{d}}\left|\nabla_{p} g\right|^{2} \mu_{\infty} \mathrm{d} p, \quad C_{\mathrm{p}}>0 .
$$

Note that in the fermionic case, $\kappa=-1$, this lemma indeed holds more generally for any $\theta \in \mathbb{R}$.

Proof. Let $\mathcal{A}$ be defined by $\mathcal{A}=-\ln \mu_{\infty}$. Inequality (3.6) in [1] shows that if $\mathcal{A}$ is uniformly convex then a Poincaré inequality with measure $\mu_{\infty} / \rho_{\infty}$ (where we divide by $\rho_{\infty}$ for normalization purposes) holds. Moreover, Theorem 3.3 in [1] allows to take into account also $L^{\infty}$ perturbations of $\mathcal{A}$. Thus we wish to decompose

$$
\mathcal{A}=\mathcal{A}_{1}+\mathcal{A}_{2}
$$

where $\mathcal{A}_{1}(p)$ is uniformly convex and $\mathcal{A}_{2}(p)$ is an $L^{\infty}$ perturbation. To this end note that $\mathcal{A}$ is given by

$$
\begin{aligned}
\mathcal{A} & =-\log \left(\frac{e^{|p|^{2} / 2+\theta}}{\left(e^{|p|^{2} / 2+\theta}-\kappa\right)^{2}}\right)=\frac{|p|^{2}}{2}+\theta-2 \log \left(\frac{e^{|p|^{2} / 2+\theta}}{e^{|p|^{2} / 2+\theta}-\kappa}\right) \\
& =\frac{|p|^{2}}{2}+\theta+2 \log \left(1-\frac{1}{\kappa e^{|p|^{2} / 2+\theta}}\right) .
\end{aligned}
$$

We now pick $\mathcal{A}_{1}=|p|^{2} / 2$ and $\mathcal{A}_{2}$ to be the rest of the terms appearing on the right hand side. Then $\mathcal{A}_{2}$ is bounded if $\kappa=-1$, or if $\kappa=1$ and $\theta>0$, which concludes the proof.

With the above lemma in hand we can now establish the coercivity of the linearized collision operator. From now on we will denote by $K, K_{1}, \ldots$ generic (positive) constants, to be used several times in different proofs, whereas the constants $C_{1}, C_{2}, \ldots$ will be fixed throughout the work in order to be able to track their appearance.

Lemma 2.2. For $\theta>0$ there exists a $\lambda>0$ such that

$$
\langle L(g), g\rangle_{L_{p}^{2}} \leq-\lambda\|g-\Pi(g)\|_{\Lambda_{p}}^{2}, \quad \forall g \in \Lambda_{p} .
$$

The coercivity property (in $p \in \mathbb{R}^{d}$ ) of the operator $L$ is indeed an essential requirement to establish our main result.

Proof. We start with

$$
\begin{aligned}
\langle L(g), g\rangle_{L_{p}^{2}} & =-\rho_{\infty} \int\left|\nabla_{p}\left(\frac{g}{\sqrt{\mu_{\infty}}}\right)\right|^{2} \frac{\mu_{\infty}}{\rho_{\infty}} \mathrm{d} p \\
& \leq-C_{\mathrm{p}}\left(\int g^{2} \mathrm{~d} p-\frac{1}{\rho_{\infty}}\left(\int \sqrt{\mu_{\infty}} g \mathrm{~d} p\right)^{2}\right) \\
& \leq-K_{1} \int\left(g-\frac{\sqrt{\mu_{\infty}}}{\rho_{\infty}} \int \sqrt{\mu_{\infty}} g \mathrm{~d} p\right)^{2} \mathrm{~d} p=-K_{1}\|g-\Pi(g)\|_{L_{p}^{2}}^{2},
\end{aligned}
$$


for some $K_{1}>0$, where we used the fact that the measure $\mu_{\infty} / \rho_{\infty}$ satisfies a Poincaré inequality due to the previous lemma. Now to improve on the $L_{p}^{2}$-norm we use

$$
\begin{aligned}
\langle L(g), g\rangle_{L_{p}^{2}} & =-\int\left(\nabla_{p}(g-\Pi(g))+\frac{p}{2} \eta_{\infty}(g-\Pi(g))\right)^{2} \mathrm{~d} p \\
& \leq-K_{2}\|g-\Pi(g)\|_{\Lambda_{p}}^{2}+K_{3}\|g-\Pi(g)\|_{L_{p}^{2}}^{2} .
\end{aligned}
$$

Adding the two inequalities above (multiplied by appropriate constants) finishes the proof.

We get a similar result for the derivatives w.r.t. $p \in \mathbb{R}^{d}$.

Lemma 2.3. Let $\theta>0$, then there exist positive constants $C_{1}$ and $C_{2}$, such that for any $g \in L_{p}^{2}$ with $\nabla_{p} g \in \Lambda_{p}$,

$$
\left\langle\nabla_{p} L(g), \nabla_{p} g\right\rangle_{L_{p}^{2}} \leq-C_{1}\left\|\nabla_{p} g\right\|_{\Lambda_{p}}^{2}+C_{2}\|g\|_{L_{p}^{2}}^{2} .
$$

Proof. A lengthy calculation yields

$$
\begin{aligned}
\left\langle\nabla_{p} L(g), \nabla_{p} g\right\rangle_{L_{p}^{2}}= & \int-\left(\Delta_{p} g\right)^{2}+\left|\nabla_{p} g\right|^{2}\left(\frac{d}{2} \eta_{\infty}-\frac{|p|^{2}}{4}-2 \kappa \mu_{\infty}|p|^{2}\right) \mathrm{d} p \\
& +\int \frac{g^{2}}{2}\left(d^{2} \kappa \mu_{\infty}+\frac{d}{2}+4 \kappa d \mu_{\infty}\right) \mathrm{d} p \\
& -\frac{\kappa}{2} \int g^{2}|p|^{2} \mu_{\infty}\left((d+10) \eta_{\infty}-2|p|^{2} \mu_{\infty} \eta_{\infty}\right) \mathrm{d} p .
\end{aligned}
$$

The last integral on the right hand side is dominated by the $L_{p}^{2}$-norm, since $\mu_{\infty}$ decays exponentially fast as $|p| \rightarrow \infty$. We also have that $1 / 4+2 \kappa \mu_{\infty} \geq C>0, \forall p \in \mathbb{R}^{d}$. This obviously holds true for the Bosonic case but is also guaranteed in the fermionic situation where $f_{\infty}<1 / 2$. Thus we can estimate

$$
\left\langle\nabla_{p} L(g), \nabla_{p} g\right\rangle_{L_{p}^{2}} \leq-C_{1}\left\|\nabla_{p} g\right\|_{\Lambda_{p}}^{2}+\frac{d}{2} \int \eta_{\infty}\left|\nabla_{p} g\right|^{2} \mathrm{~d} p+C_{2}\|g\|_{L_{p}^{2}}^{2}
$$

and a classical interpolation argument applied to the second term on the right hand side yields the assertion of the lemma (with different constants $C_{1}, C_{2}$ ).

Finally we need the following technical lemma.

Lemma 2.4. For $g, h \in \Lambda_{p}$ it holds that

$$
\langle L(h), g\rangle_{L_{p}^{2}} \leq C_{3}\|g\|_{\Lambda_{p}}\|h\|_{\Lambda_{p}}, \quad C_{3}>0 .
$$

The combination of Lemma 2.2 and Lemma 2.4 induces the particular choice of $\Lambda_{p}$ and its corresponding norm.

Proof. We first note that

$$
\begin{aligned}
& \|g\|_{\Lambda_{p}}\|h\|_{\Lambda_{p}} \\
= & \left(\left\|\nabla_{p} g\right\|_{L_{p}^{2}}^{2}+\left\|p \eta_{\infty} g\right\|_{L_{p}^{2}}^{2}\right)^{\frac{1}{2}}\left(\left\|\nabla_{p} h\right\|_{L_{p}^{2}}^{2}+\left\|p \eta_{\infty} h\right\|_{L_{p}^{2}}^{2}\right)^{\frac{1}{2}} \\
\geq & \frac{1}{C_{3}}\left(\left\|\nabla_{p} g\right\|_{L_{p}^{2}}^{2}+\int\left(1+|p|^{2}\right) \eta_{\infty}^{2} g^{2} \mathrm{~d} p\right)^{\frac{1}{2}}\left(\left\|\nabla_{p} h\right\|_{L_{p}^{2}}^{2}+\int\left(1+|p|^{2}\right) \eta_{\infty}^{2} h^{2} \mathrm{~d} p\right)^{\frac{1}{2}}
\end{aligned}
$$


for some $C_{3}>0$, since the $\Lambda_{p}$-norm dominates the $L_{p}^{2}$-norm. Using the following simple algebraic estimate,

$$
\left(\left(a^{2}+b^{2}\right)\left(c^{2}+d^{2}\right)\right)^{\frac{1}{2}} \geq a c+b d,
$$

(with $a, b, c, d \in \mathbb{R}$ ) we further obtain

$$
C_{3}\|g\|_{\Lambda_{p}}\|h\|_{\Lambda_{p}} \geq\left\|\nabla_{p} g\right\|_{L_{p}^{2}}\left\|\nabla_{p} h\right\|_{L_{p}^{2}}+\left(\int\left(1+|p|^{2}\right) \eta_{\infty}^{2} g^{2} \mathrm{~d} p \int\left(1+|p|^{2}\right) \eta_{\infty}^{2} h^{2} \mathrm{~d} p\right)^{\frac{1}{2}} .
$$

The proof then follows by applying the Cauchy-Schwarz inequality to both terms on the right hand side and integrating by parts in the first one.

\section{Convergence for the linear model and proof of Theorem 1.1}

Now we are able to establish the long time asymptotics for the linearized equation, which eventually will be translated also to the nonlinear model (1.3) in the perturbative setting.

Proposition 3.1. Consider the linearized Fokker-Planck type equation

$$
\partial_{t} g+\left(1+2 \sigma \kappa f_{\infty}\right) p \cdot \nabla_{x} g=L(g),
$$

with $L$ given by (1.8) and $\theta>0$. Moreover, if $\kappa=\sigma=1$, assume in addition that $\theta>\theta^{*}$. Let the initial data $g_{0} \in H^{k}\left(\mathbb{T}^{d} \times \mathbb{R}^{d}\right)$, for $k \in \mathbb{N}$. Then the solution $g(t)$ exists globally in time, and

$$
\left\|g(t)-g_{\infty}\right\|_{H^{k}} \leq C e^{-\tau t}, \quad \text { with } C=C\left(\left\|g_{0}\right\|_{H^{k}}\right), \tau>0,
$$

where the global equilibrium $g_{\infty}$ is given by

$$
g_{\infty}=\left(\frac{1}{\rho_{\infty}} \iint_{\mathbb{T}^{d} \times \mathbb{R}^{d}} g_{0} \sqrt{\mu_{\infty}} \mathrm{d} p \mathrm{~d} x\right) \sqrt{\mu_{\infty}} .
$$

We want to remind the reader of the discussion of the role of $\theta^{*}$ in Rem. 1.1.

Proof. For the proof we proceed similarly to [19]. We will sketch the main ideas and stress the differences which occur due to the changes in the transport operator. Note that, since the equation is linear, we can withour restriction of generality consider the case where $g_{\infty} \equiv 0$. This can always be achieved by subtracting initially the projection onto the global equilibrium, i.e. by considering initial data $\widetilde{g}_{0}=g_{0}-g_{\infty}$.

We start with $k=1$. The main idea of the proof is to study the time evolution of a combination of derivatives w.r.t. $x$ and $p$. More precisely, we consider the functional

$$
\mathcal{F}[g(t)]:=\alpha\|g\|^{2}+\beta\left\|\nabla_{x} g\right\|^{2}+\gamma\left\|\nabla_{p} g\right\|^{2}+\delta\left\langle\nabla_{x} g, \nabla_{p} g\right\rangle,
$$

where $\|\cdot\|$ denotes the standard norm on $L^{2}\left(\mathbb{T}_{x}^{d} \times \mathbb{R}_{p}^{d}\right)$ and $\alpha, \beta, \gamma, \delta$ are some positive constants. We note that $\delta$ has to be small enough in comparison to $\beta$ and $\gamma$ such that $\mathcal{F}$ is positive and controlled from above and below by the square of the usual $H^{1}\left(\mathbb{T}_{x}^{d} \times \mathbb{R}_{p}^{d}\right)$-norm of $g$. On the other hand, $\delta$ has to be strictly positive, in order to close the argument (see below). We aim to prove that

$$
\frac{\mathrm{d}}{\mathrm{d} t} \mathcal{F}[g(t)] \leq-\tilde{C}\left(\|g\|_{\Lambda}^{2}+\left\|\nabla_{x, p} g\right\|_{\Lambda}^{2}\right), \quad \tilde{C}>0 .
$$


To this end we calculate the time derivatives of the various summands in $\mathcal{F}$. First, for the $L^{2}$-norm we have

$$
\left.\frac{\mathrm{d}}{\mathrm{d} t}\|g\|^{2}=2\langle L(g), g)\right\rangle \leq-2 \lambda\|g-\Pi(g)\|_{\Lambda}^{2},
$$

where we have used that the transport part does not contribute due to its divergence form (which is a straightforward calculation) and the assertion of Lemma 2.2.

Next the spatial derivatives evolve according to

$$
\frac{\mathrm{d}}{\mathrm{d} t}\left\|\nabla_{x} g\right\|^{2}=2\left\langle\nabla_{x} L(g), \nabla_{x} g\right\rangle \leq-2 \lambda\left\|\nabla_{x} g-\Pi\left(\nabla_{x} g\right)\right\|_{\Lambda}^{2},
$$

where we again used that the contribution from the transport term vanishes and the fact that $L$ commutes with $\nabla_{x}$, thus allowing us to apply Lemma 2.2 also to $\nabla_{x} g$.

For the derivatives w.r.t. $p$ we get some additional terms from the coefficient of the transport operator

$$
\begin{aligned}
\frac{\mathrm{d}}{\mathrm{d} t}\left\|\nabla_{p} g\right\|^{2} & =2\left\langle\nabla_{p} L(g), \nabla_{p} g\right\rangle+\left\langle\left(4 \kappa \sigma \mu_{\infty}|p|^{2}-2 \eta_{\infty}\left(1+2 \kappa \sigma f_{\infty}\right)\right) \nabla_{x} g, \nabla_{p} g\right\rangle \\
& \leq 2\left\langle\nabla_{p} L(g), \nabla_{p} g\right\rangle+K \iint\left|\nabla_{p} g\right|\left|\nabla_{x} g\right| \mathrm{d} p \mathrm{~d} x .
\end{aligned}
$$

Here we have used that $f_{\infty}$ as well as $|p|^{2} \mu_{\infty}$ are uniformly bounded in $L^{\infty}$. Now the first term on the r.h.s. is estimated by Lemma 2.3, which yields some damping for $\nabla_{p} g$ in the $\Lambda$-norm, i.e.

$$
\begin{aligned}
\frac{\mathrm{d}}{\mathrm{d} t}\left\|\nabla_{p} g\right\|^{2} & \leq-2 C_{1}\left\|\nabla_{p} g\right\|_{\Lambda}^{2}+2 C_{2}\|g\|^{2}+K \iint\left|\nabla_{p} g \| \nabla_{x} g\right| \mathrm{d} p \mathrm{~d} x \\
& \leq-K_{1}\left\|\nabla_{p} g\right\|_{\Lambda}^{2}+K_{2}\left\|\nabla_{x} g\right\|^{2}+2 C_{2}\|g\|^{2}
\end{aligned}
$$

with $K_{1}, K_{2}>0$. In order to deal with the term proportional to $\|g\|^{2}$, we split

$$
g=(g-\Pi(g))+\Pi(g)
$$

and estimate

$$
\|g\|^{2} \leq\|g-\Pi(g)\|^{2}+\|\Pi(g)\|^{2} \leq\|g-\Pi(g)\|^{2}+C_{\mathbb{T}}\left\|\nabla_{x} g\right\|^{2}, \quad C_{\mathbb{T}}>0 .
$$

In the second step, we used the classical Poincaré inequality with respect to $x \in \mathbb{T}^{d}$ and the fact that $\Pi(g)$ has zero mean on the torus, since $\iint g_{\infty} \mathrm{d} p \mathrm{~d} x=0$. Together with (3.5), this yields

$$
\frac{\mathrm{d}}{\mathrm{d} t}\left\|\nabla_{p} g\right\|^{2} \leq-K_{1}\left\|\nabla_{p} g\right\|_{\Lambda}^{2}+2 C_{2}\|g-\Pi(g)\|^{2}+K_{3}\left\|\nabla_{x} g\right\|^{2},
$$

where $K_{3}=K_{2}+2 C_{2} C_{\mathbb{T}}$. Note that this is an improvement to (3.5), since the term $\|g-\Pi(g)\|^{2}$ can be controlled by adjusting $\alpha$ and having in mind (3.3).

Finally we look at the mixed derivatives w.r.t. $x$ and $p$, which evolve according to

$$
\left.\frac{\mathrm{d}}{\mathrm{d} t}\left\langle\nabla_{x} g, \nabla_{p} g\right\rangle=2\left\langle L\left(\nabla_{x} g\right), \nabla_{p} g\right\rangle-\left\langle\nabla_{x} g,\left(1+2 \sigma \kappa f_{\infty}-2 \sigma \kappa \mu_{\infty}|p|^{2}\right)\right) \nabla_{x} g\right\rangle .
$$


For the first term on the right hand side we invoke Lemma 2.4, which together with the Cauchy-Schwarz inequality in $x$ implies

$$
\left\langle L\left(\nabla_{x} g\right), \nabla_{p} g\right\rangle \leq C_{3} \eta\left\|\nabla_{x} g-\Pi\left(\nabla_{x} g\right)\right\|_{\Lambda}^{2}+C_{3} \eta^{-1}\left\|\nabla_{p} g\right\|_{\Lambda}^{2}, \quad \forall \eta>0 .
$$

The second term on the right hand side of (3.7), which stems from the transport part, generates a damping for $\left\|\nabla_{x} g\right\|$ (which the operator $L$ cannot provide since it only acts in $p$ ), provided that

$$
1+2 \kappa \sigma f_{\infty}-2 \kappa \sigma \mu_{\infty}|p|^{2} \geq K_{4}>0
$$

Assuming for the moment that (3.8) is true we obtain

$$
\frac{\mathrm{d}}{\mathrm{d} t}\left\langle\nabla_{x} g, \nabla_{p} g\right\rangle \leq 2 C_{3} \eta\left\|\nabla_{x} g-\Pi\left(\nabla_{x} g\right)\right\|_{\Lambda}^{2}+2 C_{3} \eta^{-1}\left\|\nabla_{p} g\right\|_{\Lambda}^{2}-K_{4}\left\|\nabla_{x} g\right\|^{2} .
$$

In summary, this estimate, together with (3.3), (3.4), and (3.6), yields

$$
\begin{aligned}
\frac{\mathrm{d}}{\mathrm{d} t} \mathcal{F}[g(t)] \leq & -2\left(\lambda \alpha-C_{2} \gamma\right)\|g-\Pi(g)\|_{\Lambda}^{2}-2\left(\beta \lambda-C_{3} \eta \delta\right)\left\|\nabla_{x} g-\Pi\left(\nabla_{x} g\right)\right\|_{\Lambda}^{2} \\
& -\left(K_{1} \gamma-2 C_{3} \delta \eta^{-1}\right)\left\|\nabla_{p} g\right\|_{\Lambda}^{2}-\left(K_{4} \delta-K_{3} \gamma\right)\left\|\nabla_{x} g\right\|^{2} .
\end{aligned}
$$

It remains to find coefficients $\alpha, \beta, \gamma, \delta, \eta$ in $\mathcal{F}$, such such that all "bad" terms in the above given estimates (i.e. those which come with the wrong or without sign) can be controlled and the differential inequality (3.2) holds true. This can be done analogously to Step 4 in the Proof of Theorem 1.1 in [19] and we therefore will not elaborate further on it. Note that the functional $\mathcal{F}$ then clearly induces a new norm on phase space, equivalent to $H^{1}\left(\mathbb{R}^{d} \times \mathbb{T}^{d}\right)$, via $\|g\|_{\mathcal{H}^{1}}^{2}:=\mathcal{F}[g]$.

To retain the (fundamental) damping property in the spatial derivatives coming from the evolution of the mixed term it remains to show that the constraint (3.8) holds true. We denote

$$
\Psi_{\kappa}(p ; \theta):=1+2 \kappa f_{\infty}-2 \kappa \mu_{\infty}|p|^{2} .
$$

If $\kappa=-1$ and since $\theta>0$, it is clear that $\Psi_{-1}(p ; \theta) \geq K_{4}>0$, because $\eta_{\infty} \geq 0$ in this case. For Bosons, i.e. $\kappa=1$, however the situation is more difficult. Note that

$$
\lim _{|p| \rightarrow \infty} \Psi_{1}(p ; \theta)=\lim _{|p| \rightarrow \infty}\left(\eta_{\infty}-2 \mu_{\infty}|p|^{2}\right)=1, \quad \forall \theta>0,
$$

and thus by continuity it is enough to make sure that $\Psi_{1}(p ; \theta) \neq 0, \forall p \in \mathbb{R}^{d}, \theta>\theta^{*}$. Straightforward calculations show that $\Psi_{1}(p ; \theta)$ can only be zero if

$$
\left(e^{|p|^{2} / 2+\theta}\right)^{2}-2|p|^{2} e^{|p|^{2} / 2+\theta}-1=0
$$

which implies

$$
e^{|p|^{2} / 2+\theta}=|p|^{2}+\sqrt{|p|^{4}+1} .
$$

Obviously this equality can not be true for $\theta$ larger than some critical value $\theta^{*}$. Numerical experiments suggest that this critical value is approximately $\theta^{*} \approx 0.451$. In summary one obtains the final estimate (3.2), which finishes the proof for $k=1$. 
To proceed to higher order estimates in the Sobolev index $k \in \mathbb{N}$, we observe that the proof of Lemma (2.3) can be generalized in a straightforward way to obtain

$$
\left\langle\partial_{x_{\ell}} \partial_{p_{j}} L(g), \partial_{x_{\ell}} \partial_{p_{j}} g\right\rangle_{L_{p}^{2}} \leq-C_{1, k}\left\|\partial_{x_{\ell}} \partial_{p_{j}} g\right\|_{\Lambda_{p}}^{2}+C_{2, k}\|g\|_{H_{p}^{k-1}}^{2},
$$

for any multi-indices $j, \ell$ such that $k=|j|+|\ell|,|j| \geq 1$. An induction argument in $k \in \mathbb{N}$, similar to the one given in [19, Theorem 3.1], then yields the corresponding statement in $H^{k}$. There are no additional problems due to the coefficient in the transport operator, since the terms containing the highest order derivatives of $g$ can be treated as above and the lower order terms, which contain derivatives of $\Psi_{\kappa}(p ; \theta)$, can be handled by interpolation since $\partial_{p_{j}} \Psi_{\kappa}(p ; \theta) \in L^{\infty}$. This concludes the proof. $\square$

Now we apply the result for the linearized equation to the nonlinear problem.

\section{Proof of Theorem 1.1.}

Proof. We have to show that the quadratic nonlinearity does not change the estimates obtained for the linearized equation, as long as the deviation from the equilibrium is small. The function $g=\left(f-f_{\infty}\right) \mu_{\infty}^{-1 / 2}$ solves (1.7), from which we deduce

$$
\frac{\mathrm{d}}{\mathrm{d} t}\|g\|_{\mathcal{H}^{k}}^{2}=2\langle T g, g\rangle_{\mathcal{H}^{k}}+2\langle Q(g), g\rangle_{\mathcal{H}^{k}}
$$

where $T:=L-\left(1+2 \sigma \kappa f_{\infty}\right) p \cdot \nabla_{x}$ and $L, Q$ are given in (1.8), (1.9), respectively. From the proof of Proposition 3.1 we know that

$$
\langle T g, g\rangle_{\mathcal{H}^{k}} \leq-\tilde{C}\left(\sum_{|j|+|\ell| \leq k}\left\|\partial_{x_{\ell}} \partial_{p_{j}} g\right\|_{\Lambda}^{2}\right)
$$

where $\tilde{C}$ is as in (3.2). Thus, if we can prove the following property for the nonlinear part

$$
\langle Q(g), g\rangle_{H^{k}} \leq C_{Q}\|g\|_{H^{k}}^{2}\left(\sum_{|j|+|\ell| \leq k}\left\|\partial_{x_{\ell}} \partial_{p_{j}} g\right\|_{\Lambda}\right),
$$

it follows, since $\|\cdot\|_{\mathcal{H}^{k}} \simeq\|\cdot\|_{H^{k}}$, that

$$
\begin{aligned}
& \frac{\mathrm{d}}{\mathrm{d} t}\|g\|_{\mathcal{H}^{k}}^{2} \\
\leq- & 2 \tilde{C}\left(\sum_{|j|+|\ell| \leq k}\left\|\partial_{x_{\ell}} \partial_{p_{j}} g\right\|_{\Lambda}^{2}\right)+C_{Q}\|g\|_{H^{k}}^{2}\left(\sum_{|j|+|\ell| \leq k}\left\|\partial_{x_{\ell}} \partial_{p_{j}} g\right\|_{\Lambda}\right) \\
\leq & -2 \tilde{C}\left(\sum_{|j|+|\ell| \leq k}\left\|\partial_{x_{\ell}} \partial_{p_{j}} g\right\|_{\Lambda}^{2}\right)+\epsilon C_{Q}\|g\|_{H^{k}}^{2}+C_{\epsilon} C_{Q}\|g\|_{H^{k}}^{2}\left(\sum_{|j|+|\ell| \leq k}\left\|\partial_{x_{\ell}} \partial_{p_{j}} g\right\|_{\Lambda}^{2}\right) .
\end{aligned}
$$

Now choosing $\epsilon$ small enough, such that $\epsilon C_{Q} \leq \tilde{C}$, we derive

$$
\frac{\mathrm{d}}{\mathrm{d} t}\|g\|_{\mathcal{H}^{k}}^{2} \leq-\tilde{C}\left(\sum_{|j|+|\ell| \leq k}\left\|\partial_{x_{\ell}} \partial_{p_{j}} g\right\|_{\Lambda}^{2}\right)+C^{*}\|g\|_{H^{k}}^{2}\left(\sum_{|j|+|\ell| \leq k}\left\|\partial_{x_{\ell}} \partial_{p_{j}} g\right\|_{\Lambda}^{2}\right) .
$$


This concludes the proof of Theorem 1.1 by maximum principle as long as $\left\|g_{0}\right\|_{H^{k}}$ is sufficiently small.

In order to prove (3.9), we recall that $Q(g)$ is given by

$$
Q(g)=\frac{\kappa}{\sqrt{\mu_{\infty}}}\left(\operatorname{div}_{p}\left(p \mu_{\infty} g^{2}\right)\right)-\frac{\kappa}{\sqrt{\mu_{\infty}}}\left(\sigma \mu_{\infty} p \cdot \nabla_{x}\left(g^{2}\right)\right) \equiv Q_{1}(g)-Q_{2}(g),
$$

and note that $\mu_{\infty}^{-1 / 2} \partial_{x_{\ell}} \partial_{p_{j}}\left(p \mu_{\infty}\right) \in L^{\infty}\left(\mathbb{T}_{x}^{d} \times \mathbb{R}_{p}^{d}\right)$, for all multi-indeces $\ell, j \in \mathbb{N}^{d}$. We shall now treat $Q_{1}(g)$ and $Q_{2}(g)$ separately, using that $H^{k}\left(\mathbb{R}^{d}\right) \subset L^{\infty}\left(\mathbb{R}^{d}\right)$, for $k>d / 2$, together with Leibniz' formula to differentiate $Q(g)$. It is relatively easy to see that (3.9) holds for $Q_{1}(g)$, by estimates in the spirit of (3.10) below, since the $\Lambda$ norm incorporates an additional derivate w.r.t $p \in \mathbb{R}^{d}$. The estimate for $\left\langle Q_{2}(g), g\right\rangle_{H^{k}}$ is more complicated though, since $Q_{2}$ contains a derivate w.r.t. $x \in \mathbb{T}^{d}$ that is not taken into account by the $\Lambda$-norm.

Thus we have to estimate

$$
\left\langle Q_{2}(g), g\right\rangle_{H^{k}}=-\kappa \sigma \sum_{|j|+|\ell| \leq k}\left\langle\partial_{x_{\ell}} \partial_{p_{j}}\left(\sqrt{\mu_{\infty}} p \cdot \nabla_{x}\left(g^{2}\right)\right), \partial_{x_{\ell}} \partial_{p_{j}} g\right\rangle_{L^{2}} .
$$

Since $\partial_{x_{\ell}} \partial_{p_{j}} \sqrt{\mu_{\infty}} \in L^{\infty}\left(\mathbb{T}_{x}^{d} \times \mathbb{R}_{p}^{d}\right)$, the highest order terms are of the form

$$
\left\langle\sqrt{\mu_{\infty}} p \cdot \nabla_{x} \partial_{x_{\ell}} \partial_{p_{j}}\left(g^{2}\right), \partial_{x_{\ell}} \partial_{p_{j}} g\right\rangle_{L^{2}}, \quad|j|+|\ell|=k .
$$

Moreover, because of the additional derivative w.r.t. $p$ in the $\Lambda$-norm, the most problematic terms are those where $|\ell|=k$. Denoting $\partial_{\ell} \equiv \partial_{x_{\ell}}$, we compute

$$
\begin{aligned}
\left\langle\sqrt{\mu_{\infty}} p \cdot \nabla_{x} \partial_{\ell}\left(g^{2}\right), \partial_{\ell} g\right\rangle_{L^{2}}= & 2\left\langle\sqrt{\mu_{\infty}} g p \cdot \nabla_{x} \partial_{\ell} g, \partial_{\ell} g\right\rangle_{L^{2}} \\
& +\sum_{i=1}^{d}\left\langle\sqrt{\mu_{\infty}} p_{i} \sum_{\substack{0 \leq r \leq \ell+\delta_{i} \\
0<|r|<|\ell|+1}}\left(\begin{array}{c}
\ell+\delta_{i} \\
r
\end{array}\right) \partial_{r} g \partial_{\ell+\delta_{i}-r} g, \partial_{\ell} g\right\rangle_{L^{2}},
\end{aligned}
$$

where $\delta_{i}$ denotes the $i$-th standard basis vector in $\mathbb{R}^{d}$. Using the divergence theorem, we obtain

$$
\begin{aligned}
\left\langle\sqrt{\mu_{\infty}} p \cdot \nabla_{x} \partial_{\ell}\left(g^{2}\right), \partial_{\ell} g\right\rangle_{L^{2}}= & -\left\langle\sqrt{\mu_{\infty}}\left(p \cdot \nabla_{x} g\right) \partial_{\ell} g, \partial_{\ell} g\right\rangle_{L^{2}} \\
& +\sum_{i=1}^{d}\left\langle\sqrt{\mu_{\infty}} p_{i} \sum_{\substack{0 \leq r \leq \ell+\delta_{i} \\
1 \leq|r|<|\ell|}}\left(\begin{array}{c}
\ell+\delta_{i} \\
r
\end{array}\right) \partial_{r} g \partial_{\ell+\delta_{i}-r} g, \partial_{\ell} g\right\rangle_{L^{2}} .
\end{aligned}
$$

To estimate the first term on the right hand side we note that (remember $|\ell|=k$ )

$$
\left\langle\sqrt{\mu_{\infty}}\left(p \cdot \nabla_{x} g\right) \partial_{\ell} g, \partial_{\ell} g\right\rangle_{L^{2}} \leq K_{1}\left\|\partial_{\ell} g\right\|_{L^{2}}^{2}\left\|\nabla_{x} g\right\|_{L^{\infty}} \leq K_{2}\|g\|_{H^{k}}^{2}\|g\|_{H^{k}},
$$

as soon as $\mathbb{N} \ni k>1+d / 2$ and analogously for all other appearing terms. Since

$$
\|g\|_{H^{k}} \leq K_{3} \sum_{|j|+|\ell| \leq k}\left\|\partial_{x_{\ell}} \partial_{p_{j}} g\right\|_{\Lambda}
$$

we obtain the desired estimate (3.9).

Acknowledgement. L. Neumann thanks M. Escobedo for fruitful discussions on similar quantum kinetic models. C. Sparber is thankful for the kind hospitality of the Johann Radon Institute for Applied Mathematics (RICAM). 


\section{REFERENCES}

[1] A. Arnold, P. Markowich, G. Toscani and A. Unterreiter, On convex Sobolev inequalities and the rate of convergence to equilibrium for Fokker-Planck type equations, Comm. Part. Diff. Equ., 26, 43-100, 2001.

[2] W. Bao, L. Pareschi and P. Markowich, Quantum kinetic theory: modeling and numerics for Bose-Einstein condensation, Modeling and Computational Methods for Kinetic Equations, Birkhäuser, 287-320, 2004.

[3] J.A. Carrillo, J. Rosado and F. Salvarani, 1D Nonlinear Fokker-Planck equations for Fermions and Bosons, Appl. Math. Lett., to appear.

[4] L. Desvillettes and C. Villani, On the trend to global equilibrium in spatially inhomogeneous entropy-dissipating systems: the linear Fokker-Planck equation, Comm. Pure Appl. Math., $54,1-42,2001$

[5] J. Dolbeault, Kinetic models and quantum effects: a modified Boltzmann equation for FermiDirac particles, Arch. Rational Mech. Anal., 127(2), 101-131, 1994.

[6] M. Escobedo, S. Mischler and M.A. Valle, Homogeneous Boltzmann equation in quantum relativistic kinetic theory, J. Diff. Equ. (Electronic), Monograph, 4.

[7] M. Escobedo, S. Mischler and M.A. Valle, Entropy maximisation problem for quantum and relativistic particles, Bull. Soc. Math. France 133(1), 87-120, 2005.

[8] M. Escobedo, S. Mischler and J.J. L. Velazquez, Asymptotic description of Dirac mass formation in kinetic equations for quantum particles, J. Diff. Equ., 202(2), 208-230, 2004.

[9] T.D. Frank, Classical Langevin equations for the free electron gas and blackbody radiation, J. Phys. A, 37(11), 3561-3567, 2004.

[10] T.D. Frank, Nonlinear Fokker-Planck Equations, Springer Series in Synergetics, Springer, 2005.

[11] F. Herau and F. Nier, Isotropic hypoellipticity and trend to the equilibrium for the FokkerPlanck equation with high degree potential, Arch. Rat. Mech. Anal., 171(2), 151-218, 2004.

[12] G. Kaniadakis, Generalized Boltzmann equation describing the dynamics of bosons and fermions, Phys. Lett. A, 203, 229-234, 1995.

[13] G. Kaniadakis, H-theorem and generalized entropies within the framework of nonlinear kinetics, Phys. Lett. A, 288(5-6), 283-291, 2001.

[14] G. Kaniadakis and P. Quarati, Kinetic equation for classical particles obeying an exclusion principle, Phys. Rev. E, 48(6), 4263-4270, 1993.

[15] G. Lapenta, G. Kaniadakis and P. Quarati, Stochastic evolution of systems of particles obeying an exclusion principle, Phys. A, 225(3-4), 323-335, 1996.

[16] X. Lu, A modified Boltzmann equation for Bose-Einstein particles: isotropic solutions and long-time behavior, J. Stat. Phys., 98(5-6), 1335-1394, 2000.

[17] X. Lu, On spatially homogeneous solutions of a modified Boltzmann equation for Fermi-Dirac particles, J. Stat. Phys., 105(1-2), 353-388, 2001.

[18] X. Lu and B. Wennberg, On stability and strong convergence for the spatially homogeneous Boltzmann equation for Fermi-Dirac particles, Arch. Ration. Mech. Anal., 168(1), 1-34, 2003.

[19] C. Mouhot and L. Neumann, Quantitative perturbative study of convergence to equlibrium for collisional kinetic models in the torus, Nonlinearity, 19, 969-998, 2006.

[20] L. Neumann and C. Schmeiser, Convergence to global equilibrium for a kinetic model for fermions, SIAM J. Math. Anal., 36, 1652-1663, 2005.

[21] A. Rossani and G. Kaniadakis, A generalized quasi-classical Boltzmann equation, Physica A, 277, 349-358, 2000.

[22] J. Sopik, C. Sire and P.H. Chavanis, Self-gravitating Brownian systems and bacterial populations with two or more types of particles, Phys. Rev. E, 72, 26105-26144, 2005.

[23] J. Sopik, C. Sire and P.H. Chavanis, Dynamics of the Bose-Einstein condensation: analogy with the collapse dynamics of a classical self-gravitating Brownian gas, Phys. Rev. E, 74(1), 11112-11127, 2006.

[24] E.A. Uehling and G.E. Uhlenbeck, Transport phenomena in Einstein-Bose and Fermi-Dirac gases, Phys. Rev., 43, 552-561, 1933.

[25] C. Villani, A review of mathematical topics in collisional kinetic theory, in: Handbook of Mathematical Fluid Dynamics, S. Friedlander and D. Serre (eds.), Elsevier Science, 2002.

[26] C. Villani, Hypocoercivity, preprint. arXiv: http://lanl.arxiv.org/abs/math. AP/0609050

[27] C. Villani, Hypocoercive diffusion operators, in: M. Sanz-Solé, J. Soria, J.L. Varona, and J. Verdera (eds.): Proceedings of the International Congress of Mathematicians Madrid, August $2230,2006$. 